РЕФЕРЕНТНЫЕ ЗНАЧЕНИЯ ПОКАЗАТЕЛЕЙ ФУНКЦИИ ЩИТОВИДНОЙ ЖЕЛЕЗЫ

В ПЕРВОМ ТРИМЕСТРЕ БЕРЕМЕННОСТИ И РИСК РАЗВИТИЯ ГЕСТАЦИОННОГО

САХАРНОГО ДИАБЕТА У ЖЕНЩИН САНКТ-ПЕТЕРБУРГА

() П.В. Попова ${ }^{1,2}$, Е.С. Шилова' , А.С. Ткачук' , А.В. Дронова' , А.Д. Анопова' , А.Е. Николаева , Е.Н. Гринева ${ }^{1,2}$

'Национальный медицинский исследовательский центр имени В.А.Алмазова, Санкт-Петербург

${ }^{2}$ Санкт-Петербургский государственный медицинский университет им. академика И.П. Павлова, Санкт-Петербург

${ }^{3}$ СПбГБУз Женская консультация №22, Санкт-Петербург

ОБОСНОВАНИЕ. СУбклинический гипотиреоз (СГТ) и гестационный сахарный диабет (ГСД) ассоциированы с рисками для здоровья матери и ребенка. Встречаемость СГТ зависит от популяции и принятых референтных значений тиреотропного гормона (ТТГ). Вместо универсальных фиксированных референтных значений ТТГ во время беременности в настоящее время предложено использовать нормы ТТГ, специфичные для каждой популяции и триместра беременности. Специфичные для беременных женщин Северо-Запада России нормы ТТГ в настоящее время не определены. Данные относительно взаимосвязи функции щитовидной железы и ГСД противоречивы и требуют уточнения.

ЦЕЛЬ. Определение референтных значений для уровня ТТГ и свободного тироксина (св.Т4) в первом триместре беременности уженщин, проживающих в г. Санкт-Петербурге, а также оценка взаимосвязи между тиреоидным статусом в I триместре беременности и риском развития ГСД.

МЕТОДЫ. У 503 женщин на сроке беременности до 14 нед был определен уровень ТТГ, св.Т4 и антител к тиреопероксидазе (АТ к ТПО). На сроке 24-28 нед участницам исследования был выполнен пероральный глюкозотолерантный тест (ПГТТ) для скрининга на ГСД. Оценена взаимосвязь между функцией щитовидной железы, маркерами аутоиммунного процесса в щитовидной железе и выявленным ГСД.

РЕзУЛьтАТЫ. Были определены референтные значения для ТТГ (0,07-4,40 мМЕ/л) и для св.Т4 (11,7-20,3 пмоль/л). Распространенность СГТ составила 16,9\% исходя из диагностического критерия ТТГ>2,5 мМЕ/л в I триместре и 3,8\% при использовании рассчитанного в ходе исследования референтного интервала. Гипотироксинемия встречалась у 5,3\% женщин при учете референтных значений для св. Т4, предложенных производителем наборов, и у 2,8\% женщин при использовании рассчитанных нами референтных значений св.Т4. ГСД был выявлен у 23,5\% женщин, пришедших на ПГТТ. Анализ логистической регрессии выявил связь ГСД с гипотироксинемией [ОШ=7,39; $p=0,026 ; 95 \%$ ДИ 1,27-42,93] и с повышенным уровнем АТ к ТПО [ОШ=2,02; p=0,047; 95\% ДИ 1,01-4,04], значимую после переоценки с учетом возраста и ИМТ.

ЗАКЛЮчЕНИЕ. В ходе исследования были получены референтные значения ТТГ в І триместре беременности для женщин, проживающих в Санкт-Петербурге, выявлена взаимосвязь риска ГСД с повышенным уровнем антиТПО и гипотироксинемией.

КЛЮЧЕВЫЕ СЛОВА: гестационный сахарный диабет; тиреотропный гормон; тироксин; референтные значения

\title{
FIRST TRIMESTER THYROID FUNCTION IN PREGNANT WOMEN RESIDING IN SAINT PETERSBURG (RUSSIA): REFERENCE VALUES AND RISK OF GESTATIONAL DIABETES
}

(c) Polina V. Popova ${ }^{1,2}$, Ekaterina S. Shilova', Alexandra S. Tkachuk', Alexandra V. Dronova', Anna D. Anopova', Alla E. Nikolaeva ${ }^{3}$, Elena N. Grineva ${ }^{1,2}$

${ }^{1}$ Almazov National Medical Research Centre, Saint-Petersburg, Russia ${ }^{2}$ St Petersburg Pavlov State Medical University, Saint-Petersburg, Russia

${ }^{3}$ Obstetrics \& Gynecology Clinic № 22, Saint-Petersburg, Russia

BACKGROUND. Subclinical hypothyroidism during pregnancy and gestational diabetes mellitus (GDM) is known to be associated with maternal and child morbidity. The concept of subclinical dysfunction of the thyroid gland in pregnant women depends on the population-specific and trimester-specific reference values so fixed universal cutoff concentrations for thyroid-stimulating hormone (TSH) that were recommended earlier now are put under the question. Population-specific and trimester-specific reference values have not been defined for pregnant women residing in Saint Petersburg. The data concerning the association of maternal thyroid status with GDM development are controversial.

AIMS. The aim of the study was to determine the reference values of TSH and free thyroxin (fT4) in the first trimester of pregnancy in women living in St. Petersburg, and to assess the relationship between thyroid status and the risk of subsequent development of GDM. 
MATERIALS AND METHODS. The levels of TSH, fT4 and thyroid peroxidase antibodies (TPO-Ab) were analyzed in 503 pregnant women before the 14th week of gestation. The women underwent oral glucose tolerance test (OGTT) at 24-28 weeks to find out those with GDM. The association between thyroid function, thyroid autoimmunity and the risk of GDM we estimated.

RESULTS. The reference values for TSH were $0.07-4.40 \mathrm{mU} / \mathrm{L}$, and for fT4 $11.7-20.3 \mathrm{pmol} / \mathrm{L}$. The prevalence of subclinical hypothyroidism in the 503 pregnant women was $16.9 \%$ according to the diagnostic criteria of TSH> $2.5 \mathrm{mIU} / \mathrm{L}$ and $3.8 \%$ using our calculated reference interval. Hypothyroxinemia was registered in 5,3\% using reference values recommended by diagnostic tests manufacturer and in 2,8\% according to our calculated reference interval for fT4. GDM was diagnosed in $23 \%$ of women. Logistic regression analysis showed associations of hypothyroxinemia and TPO-Ab-positivity with the increased risk of GDM that remained significant after adjustments on age and body mass index (BMI) [adjusted OR (95\% $C I)=7.39$ (1.27-42.93) for hypothyroxinemia, $p=0.026$; and adjusted OR $(95 \% \mathrm{Cl})=2.02(1.01-4.04)$ for TPO-Ab-positivity, $\mathrm{p}=0.047$ ).

CONCLUSIONS. Reference intervals for first trimester TSH and fT4 have been established for pregnant women living in St. Petersburg. Hypothyroxinemia and TPO-Ab-positivity were associated with the increased risk of GDM.

KEYWORDS: gestational diabetes mellitus; thyrotropin; thyroxine; reference values

Субклинический гипотиреоз во время беременности определяется как превышение допустимых при беременности значений тиреотропного гормона (ТТГ) при нормальном уровне гормонов щитовидной железы [1, 2]. Субклинический гипотиреоз ассоциирован с рисками для здоровья матери и ребенка, включая преждевременные роды, самопроизвольные аборты и снижение показателей интеллектуального развития потомства [3, 4]. Встречаемость субклинического гипотиреоза зависит от исследуемой популяции, срока беременности, а также от принятых референтных значений для ТТГ [3]. Например, при использовании действовавших до недавнего времени фиксированных границ ТТГ (<2,5 мМЕ/л в I триместре, <3,0 мМЕ/л во II и в III триместре) встречаемость субклинического гипотиреоза может достигать 15,5\% в некоторых популяциях $[1,5]$. Универсальные фиксированные референтные значения для уровня ТТГ во время беременности в настоящее время подвергнуты сомнению Американской тиреоидологической ассоциацией [4]. В случае расширения границ нормы ТТГ до специфичных для популяции и триместра значений, распространенность субклинического гипотиреоза будет в пределах 2-4\% [3, 6], что, очевидно, снизит лекарственную, финансовую и психологическую нагрузку на беременную женщину. Специфические для беременных женщин Северо-Запада России нормы ТТГ в настоящее время нуждаются в определении.

По данным Международной федерации диабета, у 16,9\% женщин имеет место гипергликемия во время беременности, причем у 85\% из них гипергликемия обусловлена гестационным сахарным диабетом (ГСД) [7]. ГСД ассоциирован с высоким риском неблагоприятных неонатальных исходов [8]. Данные относительно взаимосвязи функции щитовидной железы и ГСД на данный момент противоречивы и требуют уточнения $[2,9,10]$. Известно, что манифестный и субклинический гипотиреоз ассоциированы с инсулинорезистентностью и могут способствовать созданию условий для развития ГСД у беременных женщин с данными патологиями $[9,10]$. В то же время в работе Cleary-Goldman и соавт., включавшей более 10000 беременных женщин, взаимосвязь между субклиническим гипотиреозом и риском развития ГСД опровергается [11].

\section{ЦЕЛЬ}

Целью данного исследования являются определение референтных значений для уровня ТТГ и св.Т4 В I триместре беременности у женщин, проживающих в г. Санкт-Петербурге, а также оценка взаимосвязи между тиреоидным статусом женщины в I триместре беременности и риском развития у нее ГСД.

\section{МЕТОДЫ}

\section{Дизайн исследования}

Исследование было выполнено на базе института эндокринологии ФГБУ «НМИЦ им. В. А. Алмазова» Минздрава России и СПбГБУЗ «Женская консультация №22» г. Санкт-Петербурга в период с марта 2012 г. по апрель 2017 г. В исследование были включены 503 женщины, поставленные на учет по беременности до 14-й недели и давшие согласие на проведение перорального глюкозотолерантного теста (ПГТТ) при сроке беременности 24-28 нед.

\section{Критерии соответствия}

Критериями исключения из исследования являлись: наличие сахарного диабета 1-го или 2-го типа и других заболеваний, влияющих на метаболизм углеводов, и отказ пациентки от участия в исследовании. При расчете референтных значений ТТГ и св. Т4 критериями исключения также были диагностированные заболевания щитовидной железы в анамнезе, многоплодная беременность, внутриутробная гибель плода и прием препаратов, влияющих на функцию щитовидной железы.

Условия проведения

В ФГБУ НМИЦ им. В. А. Алмазова обращались женщины из всех районов Санкт-Петербурга.

Продолжительность исследования

Исследование было выполнено в период с марта 2012 г. по апрель 2017 г.

\section{Описание медицинского вмешательства}

При включении в исследование у каждой женщины была взята и заморожена сыворотка венозной крови, 
Таблица 1. Характеристика участниц исследования

\section{Характеристики участниц исследования}

Предельные значения

ТТГ, мME/ת

свТ4, пмоль/л

АТ к ТПО, МЕ/мл

Возраст, лет

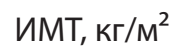

Срок беременности на момент забора крови, нед

Частота невынашивания беременности за время исследования, \% (количество)

Частота невынашивания беременности в анамнезе, \% (количество)
1 эпизод
2 эпизода
З эпизода

(минимум - макси-

мум)

$\mathrm{M} \pm \mathrm{SD}$

0,01-11,48

9,38-58,52

0,00-611,00

19-46

15,04-48,86

$5,00-14,00$

$$
\begin{gathered}
1,61 \pm 1,34 \\
15,14 \pm 3,02 \\
28,06 \pm 73,72 \\
29,43 \pm 4,70 \\
23,82 \pm 4,97 \\
10,33 \pm 2.33 \\
1,40(7)
\end{gathered}
$$

Примечание: ИМТ - индекс массы тела; ТТГ - тиреотропный гормон; св.Т4 - свободный тироксин; АТ к ТПО - антитела к тиреопероксидазе.

которую хранили при температуре $-70^{\circ} \mathrm{C}$ до выполнения анализов. Уровень ТТГ, свободного Т4 (св.Т4) и антител к тиреопероксидазе в сыворотке был определен в марте 2017 г. Уровень антиТПО выше установленных производителем наборов референтных значений (>34 ME/мл) расценивался как повышенный. На сроке 24-28 нед каждой женщине было предложено выполнить ПГТТ для скрининга на предмет ГСД. В ходе проведения ПГТТ определялся уровень глюкозы в плазме натощак, через 1 и 2 ч после приема 75 г глюкозы.

Оценивалась взаимосвязь между функцией щитовидной железы (ТТГ, св.Т4), маркерами аутоиммунного процесса в щитовидной железе (антиТПО) и выявленным ГСД. Субклиническим гипотиреозом считали изолированное повышение уровня ТТГ (в пределах до 10 мMЕ/л) при нормальном уровне св.Т4, манифестным гипотиреозом - повышение уровня ТТГ > 10 мМЕ/л и снижение уровня св.Т4, изолированной гипотироксинемией - уровень св.Т4 ниже референтных значений при нормальном уровне ТТГ, тиреотоксикозом - снижение уровня ТТГ ниже референтных значений $[1,2,4]$. Проводилась оценка распространенности нарушений тиреоидного статуса в зависимости от принятых референтных значений. Для определения «нормальных» уровней ТТГ и св.Т4 использованы: 1) фиксированные значения ТТГ, рекомендованные Европейской тиреоидологической ассоциацией для I триместра беременности: 0,1-2,5 мME/л [1]; 2) референтные значения, предложенные производителем наборов (Рош Диагностикс ГмбХ) для определения св.Т4 (12-22 пмоль/л); 3) референтные значения для ТТГ и св.Т4, рассчитанные в ходе исследования на основании распределения полученных результатов женщин с уровнем антиТПО $\leq 34$ МЕ/мл в пределах от 2,5 до 97,5 процентилей.

\section{Основной исход исследования}

Основным исходом исследования считали наличие или отсутствие ГСД.

\section{Анализ в подгруппах}

По результатам ПГТТ были сформированы две группы: группа ГСД и группа женщин без нарушения углеводно- го обмена. Диагноз ГСД, по данным ПГТТ, для инициации терапии до января 2013 г. ставили на основании критериев ВОЗ (глюкоза натощак $\geq 7,0$ ммоль/л и/или через 2 ч $\geq 7,8$ ммоль/л) [12], а с января 2013 г. - согласно рекомендациям Российского национального консенсуса [13] (глюкоза натощак $\geq 5,1$ ммоль/л, и/или через 1 ч $\geq 10,0$ ммоль/л, и/или через 2 ч $\geq 8,5$ ммоль/л). Женщины с выявленным ГСД проходили дальнейшее наблюдение и лечение в Перинатальном центре НМИЦ им. В.А. Алмазова. Диагноз ГСД по данным ПГТТ для анализа результатов и статистической обработки был ретроспективно определен по новым Российским критериям для всех беременных.

Этическая экспертиза

Протокол исследования был одобрен этическим комитетом НМИЦ им. В.А. Алмазова (№119 от 13.07.15).

\section{Статистический анализ}

Статистическую обработку данных проводили с использованием статистической программы SPSS 22.0 («SPSS Inc.», США). Данные представлены в виде $\mathrm{M} \pm \mathrm{SD}$, где M - среднее значение, SD - стандартное отклонение. В табл. 1 также представлены предельные значения (минимум и максимум). Для сравнения распределения качественных признаков использовали критерий $X^{2}$. Для оценки отличий количественных признаков между группами применяли критерий Стьюдента. Различия считали достоверными при р<0,05. Для оценки взаимосвязи между параметрами тиреоидного статуса и последующего выявления ГСД был использован метод бинарной логистической регрессии. Зависимым показателем было наличие или отсутствие ГСД.

\section{РЕЗУЛЬТАТЫ}

Объекты (участники) исследования

Всего были обследованы 503 женщины, основные характеристики которых представлены в табл. 1. Средний возраст обследованных составил 29,4 лет, средний срок беременности на момент забора крови для определения тиреоидных гормонов - 10 недель 
Таблица 2. Расчет референтных значений ТТГ и свободного тироксина в I триместре беременности

\begin{tabular}{lcccccc}
\hline $\begin{array}{c}\text { Исследуемый } \\
\text { гормон }\end{array}$ & $\begin{array}{c}\text { Среднее } \\
\text { значение }\end{array}$ & $\begin{array}{c}\mathbf{2 , 5} \\
\text { процентиль }\end{array}$ & $\begin{array}{c}\mathbf{5} \\
\text { процентиль }\end{array}$ & Медиана & $\begin{array}{c}\mathbf{9 5} \\
\text { процентиль }\end{array}$ & $\begin{array}{c}\mathbf{9 7 , 5} \\
\text { процентиль }\end{array}$ \\
\hline ТТГ, мМЕ/л & $1,49 \pm 1,05$ & 0,07 & 1,16 & 1,34 & 3,51 & 4,40 \\
свТ4, пмоль/л & $15,15 \pm 3,07$ & 11,74 & 12,16 & 14,77 & 18,96 & 20,28 \\
\hline
\end{tabular}

Примечание: ТТГ - тиреотропный гормон; свТ4 - свободный тироксин

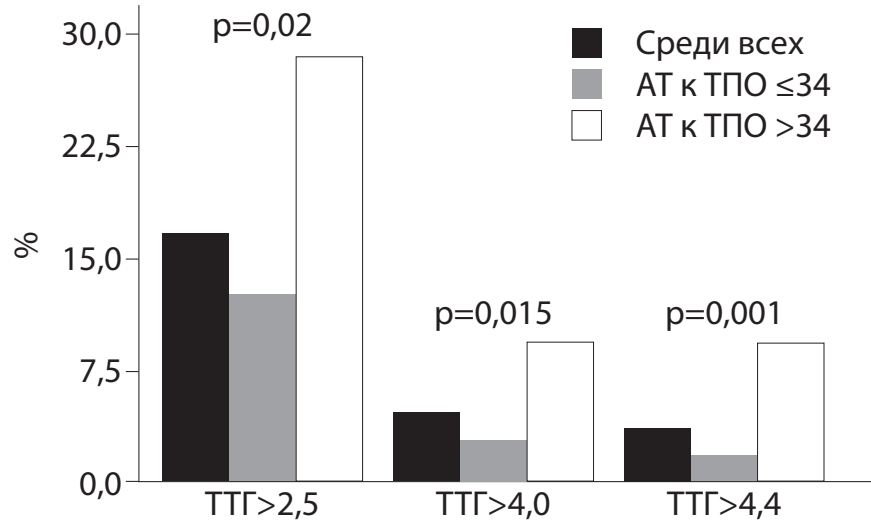

Рис. 1. Частота выявления субклинического гипотиреоза в зависимости от использованных референтных значений ТТГ и уровня антител к тиреопероксидазе.

Примечание: ТТГ - тиреотропный гормон; св.Т4 - свободный тироксин АТ к ТПО - антитела к тиреопероксидазе.

\section{Основные результаты исследования}

После исключения женщин с повышенным уровнем антитПО (N=65) или в отсутствие данных об уровне антиТПО (N=9), многоплодной беременностью $(\mathrm{N}=5)$, невынашиванием текущей беременности $(\mathrm{N}=7)$ и выявленными ранее заболеваниями щитовидной железы (N=7) были определены референтные значения для ТТГ (0,07-4,40 мМЕ/л) и для свТ4 (11,7-20,3 пмоль/л) (табл. 2).

Распространенность субклинического гипотиреоза (СГТ) среди 503 беременных женщин составила 16,9\%, исходя из диагностического критерия ТТГ>2,5 мМЕ/л В I триместре и 3,8\% при использовании рассчитанного в ходе исследования референтного интервала. При этом СГТ, вне зависимости от выбранных критериев, достоверно чаще встречался среди женщин с повышенным уровнем АТ к ТПО, рис. 1.

Распространенность гипотироксинемии также различалась в зависимости от принятых референтных значений: при диапазоне св.Т4 12-22 пмоль/л, указан- ном производителем реагентов, гипотироксинемия встречалась у 5,3\% женщин, тогда как при рассчитанной нами норме св.Т4 11,7-20,3 пмоль/л гипотироксинемия имела место у 2,8\% участниц исследования. При повышенном уровне АТ к ТПО гипотироксинемия встречалась чаще, чем при их нормальном уровне, вне зависимости от используемых референтных значений для св.Т4 (рис. 2).

ГСД был выявлен у 102 (23,5\%) женщин, пришедших на ПГТТ (N=434). Характеристики участниц исследования, пришедших на ПГТТ, представлены в табл. 3. Женщины с ГСД были старше $(30,8 \pm 4,4$ и 29,0 4 4,8 лет, $\mathrm{p}=0,001)$ и имели более высокий ИМТ по сравнению

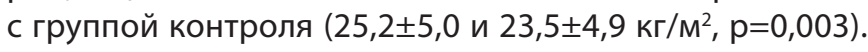
Гипотироксинемия, диагностированная с использованием рассчитанных нами критериев, встречалась достоверно чаще среди женщин с ГСД по сравнению с женщинами без нарушения углеводного обмена $(6,1 \%$ и 1,2\%, p=0,005), так же, как и повышенный уровень АТ к ТПО (18,9\% и 9,4\% соответственно, $p=0,012)$. Достоверных различий в уровнях ТТГ, св. Т4 и АТ к ТПО, а также частоте выявления субклинического и манифестного гипотиреоза между данными группами выявлено не было.

\section{Дополнительные результаты исследования}

Анализ логистической регрессии показал взаимосвязь между гипотироксинемией и риском развития ГСД, которая остается значимой после переоценки с учетом возраста и ИМТ [ОШ=7,39; $p=0,026 ; 95 \%$ ДИ 1,27-42,93]. Кроме того, была выявлена ассоциация между повышенным уровнем антиТПО и риском ГСД, также достоверная после коррекции с учетом возраста и ИМТ [ОШ=2,02; $\mathrm{p}=0,047 ; 95 \%$ ДИ 1,01-4,04]. Ассоциации между риском ГСД и уровнем ТТГ выявлено не было (рис. 3). Достоверной взаимосвязи между частотой невынашивания беременности и функцией щитовидной железы обнаружено не было.

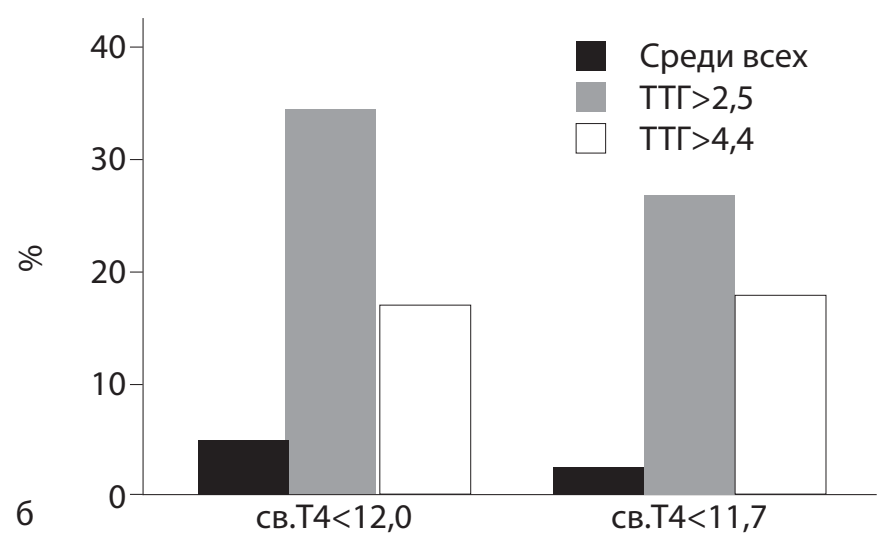

a

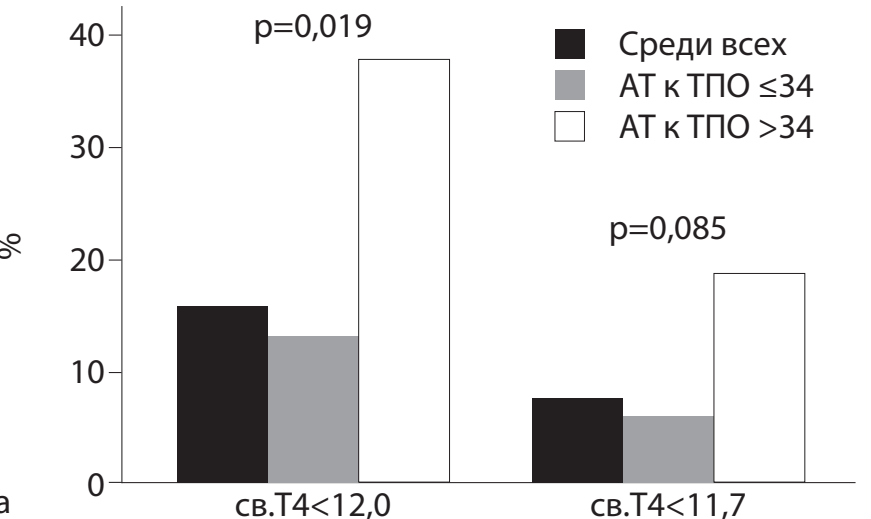

Рис. 2. Частота гипотироксинемии при использовании разных референтных значений св.Т4: а - в зависимости от наличия повышенного уровня АТ к ТПО; б - в зависимости от уровня ТТГ.

Примечание: ТТГ - тиреотропный гормон; св.Т4 - свободный тироксин; АТ к ТПО - антитела к тиреопероксидазе 
Таблица 3. Сравнительная характеристика женщин с ГСД и без ГСД

\begin{tabular}{|c|c|c|c|}
\hline Характеристика участниц исследования & ГСД $(\mathrm{N}=102)$ & Без ГСД (N=332) & $\mathbf{P}$ \\
\hline Возраст, лет & $30,8 \pm 4,4$ & $29,0 \pm 4,8$ & $p=0,001$ \\
\hline 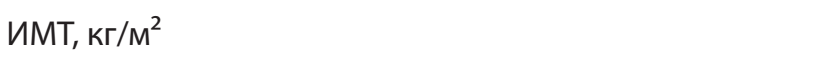 & $25,2 \pm 5,0$ & $23,5 \pm 4,9$ & $p=0,003$ \\
\hline ТТГ, мМЕ/л & $1,4 \pm 1,3$ & $1,7 \pm 1,4$ & $p=0,066$ \\
\hline Свободный тироксин (св.Т4), пмоль/л & $15,1 \pm 5,0$ & $15,3 \pm 2,3$ & $p=0,687$ \\
\hline Антитела к тиреопероксидазе (АТ к ТПО), МЕ/мл & $42,4 \pm 94,3$ & $26,6 \pm 72,8$ & $p=0,128$ \\
\hline $\begin{array}{l}\text { Частота субклинического гипотиреоза, \% } \\
\text { при ТТГ >2,5 } \\
\text { при ТТГ >4,4 }\end{array}$ & $\begin{array}{c}11,8 \\
1,0\end{array}$ & $\begin{array}{c}17,8 \\
4,5\end{array}$ & $\begin{array}{l}p=0,151 \\
p=0,097\end{array}$ \\
\hline $\begin{array}{l}\text { Частота манифестного гипотиреоза, \% } \\
\text { при ТТГ }>2,5 \\
\text { при ТТГ > }>4,4\end{array}$ & $\begin{array}{l}2,0 \\
1,0\end{array}$ & $\begin{array}{l}0,9 \\
0,6\end{array}$ & $\begin{array}{l}p=0,362 \\
p=0,668\end{array}$ \\
\hline $\begin{array}{l}\text { Частота гипотироксинемии, \% } \\
\text { при св.Т4 <12,0 } \\
\text { при св.Т4 <11,7 }\end{array}$ & $\begin{array}{l}9,2 \\
6,1\end{array}$ & $\begin{array}{l}3,3 \\
1,2\end{array}$ & $\begin{array}{l}p=0,016 \\
p=0,005\end{array}$ \\
\hline Частота выявления повышенного уровня АТ к ТПО, \% & 18,9 & 9,4 & $p=0,012$ \\
\hline
\end{tabular}

Примечание: ИМТ - индекс массы тела; ТТГ - тиреотропный гормон; св.Т4 - свободный тироксин; АТ к ТПО - антитела к тиреопероксидазе

\section{ОБСУЖДЕНИЕ}

\section{Резюме основного результата исследования}

В ходе исследования были определены референтные значения ТТГ и св. Т4 в I триместре беременности для женщин, проживающих в Санкт-Петербурге. Полученные значения превышают существующие фиксированные нормы. Выявлена взаимосвязь риска развития ГСД с повышенным уровнем антиТПО и гипотироксинемией. При этом ассоциации между риском ГСД и уровнем ТТГ, а также наличием гипотиреоза или тиреотоксикоза выявлено не было.

\section{Обсуждение основного результата исследования}

Фиксированные нормы ТТГ во время беременности подвергнуты сомнению в клинических рекомендациях Американской тиреоидологической ассоциации 2017 г. [4]. Поводом для пересмотра подхода к диагностике СГТ послужили результаты ряда исследований, показавших заметные различия в нормах ТТГ для разных популяций $[4,6,14]$. Различия в уровнях ТТГ между популяциями, вероятно, связаны с ее этническим составом, с обеспеченностью йодом и географическим расположением региона [4]. Определенные в ходе данного исследования референтные значения для ТТГ также заметно превосходят рекомендуемые во время беременности фиксированные нормы [1, 2]. Частота выявления субклинического гипотиреоза в обследованной выборке при этом сопоставима с данными других исследований при использовании как фиксированных критериев, так и высчитанных норм для данной популяции [5]. Складывается представление о заниженных нормах ТТГ для женщин, проживающих в Санкт-Петербурге.

В то же время Американская тиреоидологическая ассоциация рекомендует рассчитывать специфические для популяции референтные значения ТТГ на выборках с достаточным йодообеспечением [4]. Санкт-Петербург же относится к регионам с легким дефицитом йода [15]. По данным Д.Е. Соболевой и соавт., при обследовании 103 беременных женщин, проживающих в Санкт-Петербурге, медиана йодурии составила 117,00 мкг/л (75,00-170,90), что соответствует недостатку йода. При этом 69,8\% находились в состоянии йододефицита, имея йодурию ниже 150 мкг/л. Процент новорожденных, имеющих уровень тиреотропного гормона более

Повышенный уровень АТ к ТПО

(АТ к ТПО > 34 МЕ/мл)

Гипотироксинемия

(св.Т4<11,7 пмоль/л)

Тиреотоксикоз

(ТТГ<0,07 мMЕ/л)

Гипотиреоз

(ТТГ>4,4 мМЕ/л)

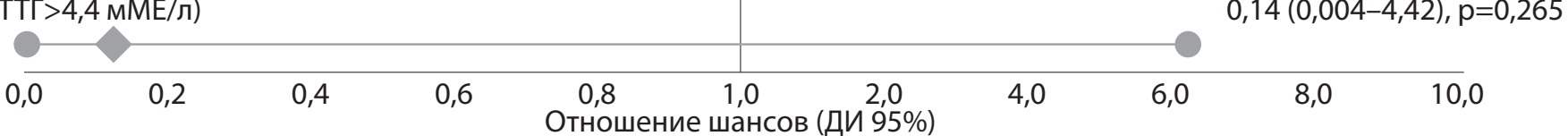

Рис. 3. Связь функции щитовидной железы и риска развития ГСД по данным регрессионного анализа.

Примечание: ТТГ - тиреотропный гормон; св.Т4 - свободный тироксин; АТ к ТПО - антитела к тиреопероксидазе 
5 мЕД/л, за 2013-2014 гг. составил 6,9\%, что также соответствует легкому дефициту йода в регионе [15].

Обращает на себя внимание высокая частота выявления ГСД по результатам ПГТТ в обследованной выборке (23\%), что превышает встречаемость ГСД по данным НАРО (17,8\%) [8] и исследования европейской популяции (12,4\%) [16]. Возможно, это обусловлено тем, что на ПГТТ чаще приходили женщины с факторами риска ГСД. В связи с тем, что до конца 2012 г. универсальный скрининг на ГСД не был официально рекомендован в России, и внедрение его в Санкт-Петербурге продолжается до сих пор, женщины без факторов риска чаще отказывались от выполнения ПГТТ. Поэтому, хотя мы приглашали на ПГТТ всех женщин, вставших на учет в ЖК №22 до 14 нед гестации, полученные результаты не следует экстраполировать на всю популяцию и делать вывод о распространенности ГСД в Санкт-Петербурге.

Механизм взаимосвязи между аутоиммунными процессами в щитовидной железе, функцией щитовидной железы и риском ГСД в настоящее время не изучен [17]. При манифестирующем и субклиническом гипотиреозе доказано повышение резистентности периферических тканей к инсулину, а также повышение концентрации инсулина в крови [11]. В соответствии с этим можно было бы ожидать увеличения риска ГСД у женщин со сниженной функцией щитовидной железы, что и находит подтверждение в литературе [9, 10]. В крупном исследовании Tudela и соавт. была выявлена прямая корреляция между уровнем ТТГ и риском ГСД, сохранившая свою достоверность при учете в расчетах возраста, ИМТ и этнической принадлежности. Риск ГСД у женщин с субклиническим гипотиреозом также был достоверно выше, но при учете факторов риска ГСД уровень достоверности оказался несколько ниже допустимого значения $(p=0,056)$ [9]. Cleary-Goldman и соавт. при обследовании более чем 10000 беременных женщин, напротив, не зафиксировали корреляции между субклиническим гипотиреозом в I триместре беременности и риском развития ГСД, что, вероятно, связано с меньшим этническим разнообразием и меньшим уровнем антиТПО в исследуемой выборке по сравнению с работой Tudela [11]. Взаимосвязь риска ГСД с уровнем ТТГ в нашем исследовании подтверждена не была.

В нескольких исследованиях были получены данные об ассоциации гипотироксинемии среди эутиреоидных женщин во II и III триместрах с риском ГСД [11]. Однако взаимосвязь гипотироксинемии в I триместре беременности и риска ГСД не подтверждена в некоторых работах $[10,11]$. Выявленная в нашем исследовании ассоциация между гипотироксинемией и риском ГСД согласуется с результатами, полученными Yang и соавт. и Oguz и соавт. в своих работах $[18,19]$. Противоречивость данных литературы свидетельствует о необходимости дальнейшего изучения прогностической значимости уровня св.T4 для оценки риска развития ГСД.

Результаты исследований ассоциации аутоиммунного процесса в щитовидной железе и риска ГСД также неоднозначны $[17,20]$. Выявленная нами взаимосвязь между повышенным уровнем антиТПО и риском ГСД соответствует данным других исследований $[17,20]$. Но мета-анализ на основе 20 исследований, включивших 34566 участников, выявил лишь слабую ассоциацию между уровнем антиТПО и риском ГСД (ОР=1,12; 95\% ДИ 1,03-1,22) [17]. При этом взаимосвязь между сниженной функцией щитовидной железы и риском ГСД у женщин с повышенным уровнем анти-ТПО оказалась значительно сильнее (OP=1,35, 95\% ДИ 1,06-1,71) [17]. В других исследованиях риск ГСД у женщин с повышенным уровнем ТТГ и антиТПО оценивается еще выше - в 3,2 раза по сравнению с женщинами с эутиреоидным состоянием без признаков аутоиммунного процесса в щитовидной железе [20]. В нашем исследовании такая корреляция зафиксирована не была, возможно, в связи с ограниченным размером исследуемой выборки.

\section{Преимущества и ограничения исследования}

В нашем исследовании впервые рассчитаны референтные значения ТТГ и св.Т4 в І триместре беременности для женщин, проживающих в Северо-Западном регионе России. В то же время малый объем исследуемой выборки и отсутствие определения йодного статуса (медианы йодурии) обследованных женщин являются ограничивающими факторами, препятствующими применению этих значений для всей популяции женщин данного региона.

\section{ЗАКЛЮЧЕНИЕ}

Нормы уровня ТТГ значительно различаются в зависимости от популяции и срока беременности. Референтные значения ТТГ для разных регионов России в настоящее время не определены. В ходе нашего исследования были получены референтные значения ТТГ в I триместре беременности для женщин, проживающих в Санкт-Петербурге (0,07-4,40 мME/л). Рассчитанные нами границы ТТГ значительно превышают существующие фиксированные нормы, из чего можно сделать вывод о необходимости проведения дальнейших исследований на большей выборке пациентов и в других регионах России.

Взаимосвязь аутоиммунных процессов в щитовидной железе и риска развития ГСД недостаточно изучена, а данные относительно существования этой взаимосвязи противоречивы. В нашем исследовании выявлена взаимосвязь риска ГСД с повышенным уровнем антиТПО и гипотироксинемией. Ассоциация между уровнем ТТГ и риском ГСД подтверждена не была. Чтобы определить прогностическую значимость тиреоидного статуса и маркеров аутоиммунного процесса для риска ГСД, требуются дальнейшие исследования на большей выборке пациентов.

\section{ДОПОЛНИТЕЛЬНАЯ ИНФОРМАЦИЯ}

Источник финансирования. Исследование проведено при финансовой поддержке Российского научного фонда (проект № 15-14-30012).

Конфликт интересов. Авторы декларируют отсутствие явных и потенциальных конфликтов интересов, связанных с публикацией настоящей статьи.

Участие авторов. Попова П.В - дизайн исследования, статистическая обработка и анализ результатов, написание рукописи; Шилова Е.С. - сбор данных, статистическая обработка результатов, написание текста; Ткачук А.С. - сбор данных, консультирование пациентов; Дронова А.В. - сбор данных, консультирование пациентов; Анопова А.Д. - сбор данных; Николаева А.Е. - сбор данных; Кутуева Ф.Р. - сбор данных; Гринева Е.Н. - организация исследования, анализ результатов. 


\section{СПИСОК ЛИТЕРАТУРЫ | REFERENCES}

1. Lazarus J, Brown RS, Daumerie C, et al. 2014 European thyroid association guidelines for the management of subclinical hypothyroidism in pregnancy and in children. Eur Thyroid J. 2014;3(2):76-94 doi: 10.1159/000362597

2. Фадеев В.В. По материалам клинических рекомендаций по диагностике и лечению заболеваний щитовидной железы во время беременности и в послеродовом периоде Американской тиреоидной ассоциации // Клиническая и экспериментальная миреоидология. - 2012. - Т. 8. - №1. - C. 7-18. [Fadeyev W. Guidelines of the American Thyroid Association for the Diagnosis and Management of Thyroid Disease During Pregnancy and Postpartum. Clinical and experimental thyroidology. 2012;8(1):7-18. (In Russ.)] doi: 10.14341/ket2012817-18

3. Maraka S, Ospina NM, O'Keeffe DT, et al. Subclinical Hypothyroidism in Pregnancy: A Systematic Review and Meta-Analysis. Thyroid. 2016;26(4):580-590. doi: 10.1089/thy.2015.0418

4. Alexander EK, Pearce EN, Brent GA, et al. 2017 Guidelines of the American Thyroid Association for the Diagnosis and Management of Thyroid Disease During Pregnancy and the Postpartum. Thyroid. 2017;27(3):315-389. doi: 10.1089/thy.2016.0457

5. Blatt AJ, Nakamoto JM, Kaufman HW. National status of testing for hypothyroidism during pregnancy and postpartum. J Clin Endocrinol Metab. 2012;97(3):777-784. doi: 10.1210/jc.2011-2038

6. Li C, Shan Z, Mao J, et al. Assessment of thyroid function during first-trimester pregnancy: what is the rational upper limit of serum TSH during the first trimester in Chinese pregnant women? J Clin Endocrinol Metab. 2014;99(1):73-79. doi: 10.1210/jc.2013-1674

7. Guariguata L, Linnenkamp U, Beagley J, et al. Global estimates of the prevalence of hyperglycaemia in pregnancy. Diabetes Res Clin Pract. 2014;103(2):176-185. doi: 10.1016/j.diabres.2013.11.003

8. Group HSCR, Metzger BE, Lowe LP, et al. Hyperglycemia and adverse pregnancy outcomes. N Engl J Med. 2008;358(19):1991-2002. doi: 10.1056/NEJMoa0707943

9. Tudela CM, Casey BM, McIntire DD, Cunningham FG. Relationship of subclinical thyroid disease to the incidence of gestational diabetes. Obstet Gynecol. 2012;1 19(5):983-988. doi: 10.1097/AOG.0b013e318250aeeb

10. Gorar S, Abanonu GB, Uysal A, et al. Comparison of thyroid function tests and blood count in pregnant women with versus without gestational diabetes mellitus. J Obstet Gynaecol Res. 2017;43(5):848-854. doi: $10.1111 /$ jog. 13280

11. Cleary-Goldman J, Malone FD, Lambert-Messerlian G, et al. Maternal thyroid hypofunction and pregnancy outcome. Obstet Gynecol. 2008;112(1):85-92. doi: 10.1097/AOG.0b013e3181788dd7
12. Эндокринология: начиональное руководство. / Под ред. Дедова И.И., Мельниченко Г.А. - М.: ГЭОТАР-Медиа; 2008. [Dedov II, Mel'nichenko GA, editors. Endokrinologiya: natsional'noe rukovodstvo. Moscow: GEOTAR-Media; 2008. (In Russ.)]

13. Дедов И.И., Краснопольский В.И., Сухих Г.Т. Российский национальный консенсус «Гестационный сахарный диабет: диагностика, лечение, послеродовое наблюдение» // Caхарный диабет. - 2012. - T. 15. - №4. - C. 4-10. [Dedov II, Krasnopol'skiy VI, Sukhikh GT. Russian National Consensus Statement on gestational diabetes: diagnostics, treatment and postnatal care. Diabetes Mellitus. 2012;15(4):4-10. (In Russ.)] doi: 10.14341/2072-0351-5531

14. Gilbert RM, Hadlow NC, Walsh JP, et al. Assessment of thyroid function during pregnancy: first-trimester (weeks 9-13) reference intervals derived from Western Australian women. Med J Aust. 2008;189(5):250-253.

15. Соболева Д.Е., Дора С.В., Волкова А.Р., и др. Йодообеспечение беременных женщин Санкт-Петербурга. Эффективность профилактики развития йододефицитных заболеваний в группе риска // Проблемы женского здоровья. - 2015. - T. 10. - №2. C. 25-31. [Soboleva DE, Dora SV, Volkova AR, et al. lodine status of pregnant women living in Saint Petersburg. The effectiveness of iodine prevention in risk group. Problemy zhenskogo zdorov'ya. 2015;10(2):25-31. (In Russ.)]

16. Avalos GE, Owens LA, Dunne F, Collaborators AD. Applying current screening tools for gestational diabetes mellitus to a European population: is it time for change? Diabetes Care. 2013;36(10):3040-3044. doi: $10.2337 / \mathrm{dc} 12-2669$

17. Yang Y, Li Q, Wang Q, Ma X. Thyroid antibodies and gestational diabetes mellitus: a meta-analysis. Fertil Steril. 2015;104(3):665-671 e663. doi: 10.1016/j.fertnstert.2015.06.003

18. Yang S, Shi FT, Leung PC, et al. Low Thyroid Hormone in Early Pregnancy Is Associated With an Increased Risk of Gestational Diabetes Mellitus. J Clin Endocrinol Metab. 2016;101(11):4237-4243. doi: 10.1210/jc.2016-1506

19. Oguz A, Tuzun D, Sahin M, et al. Frequency of isolated maternal hypothyroxinemia in women with gestational diabetes mellitus in a moderately iodine-deficient area. Gynecol Endocrinol. 2015;31(10):792-795. doi: 10.3109/09513590.2015.1054801

20. Ying H, Tang YP, Bao YR, et al. Maternal TSH level and TPOAb status in early pregnancy and their relationship to the risk of gestational diabetes mellitus. Endocrine. 2016;54(3):742-750. doi: 10.1007/s12020-016-1022-6

\section{ИНФОРМАЦИЯ ОБ АВТОРАХ [AUTHORS INFO]}

Попова Полина Викторовна, К.м.н. [Polina V. Popova, MD, PhD]; адрес:197341, Санкт-Петербург, ул. Аккуратова, д. 2. [address: 2, Akkuratova str., Saint Petersburg, 197341 Russian Federation]; ORCID: http://orcid.org/0000-0002-3697-7791; eLibrary SPIN: 1150-3432; e-mail: pvpopova@yandex.ru.

Шилова Екатерина Сергеевна, клинический ординатор [Ekaterina S. Shilova; MD, clinical resident]; ORCID: http://orcid.org/0000-0002-5225-6054; eLibrary SPIN: 9703-5970; e-mail: katia.shilova@gmail.com.

Ткачук Александра Сергеевна, н.c. [Alexandra S. Tkachuk, MD, research associate]; ORCID: http://orcid.org/0000-0002-1384-8743; eLibrary SPIN: 4109-4967; e-mail: aleksandra.tkachuk.1988@mail.ru.

Александра Владимировна Дронова, врач-эндокринолог [Alexandra V. Dronova, MD]; e-mail: aleksandra-dronova@yandex.ru.

Анна Дмитриевна Анопова, студентка [Anna D. Anopova, medical student];

ORCID: http://orcid.org/0000-0003-3107-4531; eLibrary SPIN: 4522-4054; e-mail: anchylove@mail.ru.

Алла Ехильевна Николаева, к.M.H. [Alla E. Nikolaeva, MD, PhD]; ORCID: http://orcid.org/0000-0003-3802-5468; eLibrary SPIN: 6472-0617; e-mail: alla-nikolaeva2007@yandex.ru.

Гринева Елена Николаевна, д.М.Н., профессор [Elena N. Grineva, MD, PhD, Professor]; ORCID: orcid.org/0000-0003-0042-76808; eLibrary SPIN: 2703-0841; e-mail: grineva_e@mail.ru. 


\section{ЦИТИРОВАТЬ:}

Попова П.В., Шилова Е.С., Ткачук А.С., Дронова А.В., Анопова А.Д., Николаева А.Е., Гринева Е.Н. Референтные значения показателей функции щитовидной железы в первом триместре беременности и риск развития гестационного сахарного диабета у женщин Санкт-Петербурга // Сахарный диабет. - 2018. — Т. 21. — №1. — C. 34-41. doi: 10.14341/DM9283

\section{TO CITE THIS ARTICLE:}

Popova PV, Shilova ES, Tkachuk AS, Dronova AV, Anopova AD, Nikolaeva AE, Grineva EN. First trimester thyroid function in pregnant women residing in Saint Petersburg (Russia): reference values and risk of gestational diabetes. Diabetes Mellitus. 2018;21(1):34-41. doi: 10.14341/DM9283 